\title{
Tissue Hardness Measurement Sensor and Algorithm for Tumor Detection Using Light Trapping and Scattering in Silicone Waveguide
}

\author{
Su Yang and Jong-Ha Lee* \\ 1095 Dalgubeoldaero, Department of Biomedical Engineering, \\ School of Medicine, Keimyung University, South Korea \\ (Received March 1, 2017; accepted July 12, 2017)
}

Keywords: tumor detection, medical device, tissue hardness, tactile sensor, optical analysis

A novel tissue hardness measurement sensor (tactile sensor) capable of measuring the material constants of sensed objects has been fabricated and demonstrated in this study. The tactile sensor we proposed is composed of an elastic optical waveguide unit, a highresolution CCD camera unit, and an LED light source. The sensing element is formed on polydimethylsiloxane (PDMS) and illuminated along its four edges by LED light sources. The sensor operates on the principle of total internal reflection within an optical waveguide. When an object compresses the waveguide, the contact area of the waveguide deforms and causes the light to scatter. Since the scattered light is directly captured by a CCD camera, the tactile resolution of the proposed sensor is based on the resolution of the camera. Experiments were conducted to demonstrate the ability of the proposed sensing strategy in measuring Young's modulus of polymer samples within $23 \%$ error.

\section{Introdution}

Diagnosing early formation of tumors or lumps, particularly those caused by cancer, has been a challenge. It has been addressed using various techniques, such as computer tomography, ultrasonic imaging, nuclear magnetic resonance imaging, X-rays, and so on. ${ }^{(1-6)}$ Each technique has limitations, including the application of radiation to the body, which may be harmful to the body being tested. In breast cancer cases, palpation plays a primary role in detecting tumors or lumps through breast self-examination or annual clinical breast examination. ${ }^{(7)}$ The problem with this method is that it is difficult to accurately verbalize and record tactile sensations. Various methods have been proposed for measuring the characteristics of tissue. One approach attempts to determine the relative stiffness or elasticity of tissue by ultrasonic imaging techniques while vibrating the tissue at low frequencies. ${ }^{(8)}$ Another method recently proposed for measuring and imaging tissue elasticity is elastography. ${ }^{(9,10)}$ This method includes emitting ultrasonic waves along a path into the tissue and detecting an echo sequence resulting from the ultrasonic wave pulse. The tissue is then compressed and uncompressed along the path; during

*Corresponding author: e-mail: segeberg@gmail.com

http://dx.doi.org/10.18494/SAM.2018.1584 
such compression, a second pulse of ultrasonic waves is sent along the path into the tissue. The second echo sequence resulting from the second ultrasonic wave pulse is detected and then the differential displacements of the selected echo segments of the first and second echo sequences are measured. A selected echo segment of the echo sequence corresponds to a particular echo source within the tissue along the beam axis of the transducer. Time shifts in the echo segment are examined to measure the compressibility of the tissue regions. It is desirable to have the capability to investigate tissue elasticity changes, which may indicate precursors of tumors or actual tumors without subjecting the patient to radiation.

Our goal is to develop a simple and easy-to-use system that can document the properties of palpable lumps in soft tissue using a noninvasive technique. The tactile sensor we proposed is composed of an elastic optical waveguide unit, a high-resolution CCD camera unit, a LED light source unit, and a laptop computer. The sensing element is formed on polydimethylsiloxane (PDMS) and illuminated along its four edges by LED light sources. The tactile sensor operates on the principle of total internal reflection (TIR) within an optical waveguide. The light directed into the waveguide remains within it owing to the TIR generated, since the waveguide is surrounded by air having a lower refractive index than the waveguide. When an object adheres to the waveguide and compresses, the contact area of the waveguide deforms and causes the light to scatter. The normal force is detected from the integrated gray-scale values of bright pixels emitted from the deformed area of the optical waveguide. We analyze the physical basis for tactile imaging by measuring stress-strain patterns that result from lights pressed against objects. Temporal and spatial changes in the stress-strain pattern allow the detection of structures with different elastic properties and the assessment of the geometrical and mechanical parameters of these structures. The effect of some hidden anatomical features, such as lumps, on the stress distribution at the touching surface is also studied. We demonstrated that the current device permits determining the presence and location of tissue with elastic characteristics different from those of the surrounding tissue. Figure 1 shows examples of a breast self-examination method and an optical tactile elasticity imaging method. By using a tactile imager, we can quantify the tactile sensation of hidden features. The hardness of tumors can be quantified in terms of the surrounding tissue elastic properties. The tactile sensation would be more sensitive than what we feel by hand.

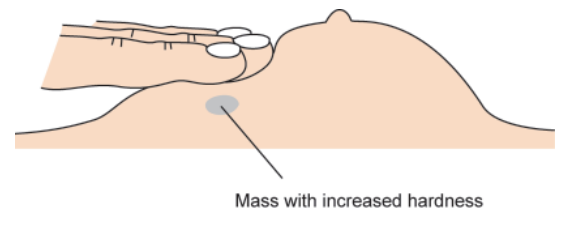

(a)

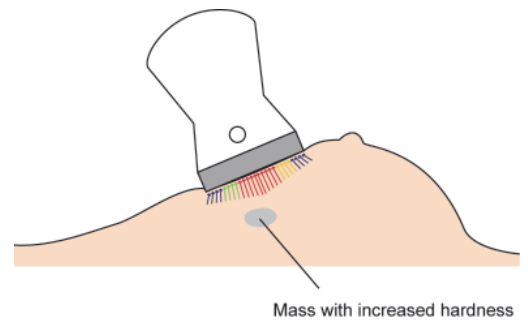

(b)

Fig. 1. (Color online) (a) Breast self-examination. (b) Tactile sensation recording by optical tactile elasticity imager. The tactile imager quantifies the hand sensation by touch. 
A method of accurately recording tactile sensations from physical exams would improve the ability to detect changes in lumps over time. We believe that tactile imaging will provide a solution to the problem of documenting the results of physical examination. It will provide more accurate and repeatable estimates of lump size than the currently available and widely used methods for breast examination, specifically clinical breast examination, ultrasound, and mammography.

\section{Tactile Imaging Sensor}

By knowing the specific features of human fingers, the basic design of the tactile sensor is introduced to satisfy the characteristics described in Sect. 2. The tactile sensor, emulating the structure of human fingers, is designed with the characteristics listed below.

1) Elastic material: PDMS is used for emulating human tissue. This was necessary to create a soft contact surface that has been proved to be effective in detecting the texture of a soft material.

2) Three-layered structure: For emulating the structure of human tissue, three types of PDMS with different elasticities (the elastic coefficient of the epidermis is the largest and that of the subcutaneous is the smallest for better sensitivity) are stacked together. The elasticity of PDMS is controlled by the base-to-catalyst ratio of PDMS.

3) Distribution of bone and nail elements: To effectively derive sensory information, parts that function as the bone and nail are situated at the base of the sensor. In the current design, a heat-resistant borosilicate glass plate is used.

4) Distributed sensor element: To emulate mechanoreceptors of the human finger, the optical method using a light reflection pattern is used. This is to obtain the spatial distribution of sensory outputs.

\subsection{Figures and tables}

The schematic view shown in Fig. 2 demonstrates the structure of the present tactile sensor. The system incorporates an elastic optical waveguide unit, a CMOS camera unit, and a computer for data acquisition and analysis. Four LED light source units are attached to the glass plate and illuminate an optical waveguide. The following section describes each unit in detail.

\subsubsection{Elastic optical waveguide}

The elastic optical waveguide is the main sensing probe of the device. It is composed of PDMS [Si( $\left.\left.\mathrm{CH}_{3}\right)_{2}\right]$. PDMS is a high-performance silicone elastomer. ${ }^{(9,10)}$ In the device, the optical waveguide should be transparent and PDMS meets this requirement. A schematic of the multilayer elastic waveguide is shown in Fig. 3.

A glass plate such as a microscope slide is placed in a shallow tray on a table. The glass must be perfectly horizontal. A He-Ne laser is reflected off the surface of the glass to produce a spot of light, $N$, on a vertical wall. Without moving the glass plate, place a PDMS on the glass. 


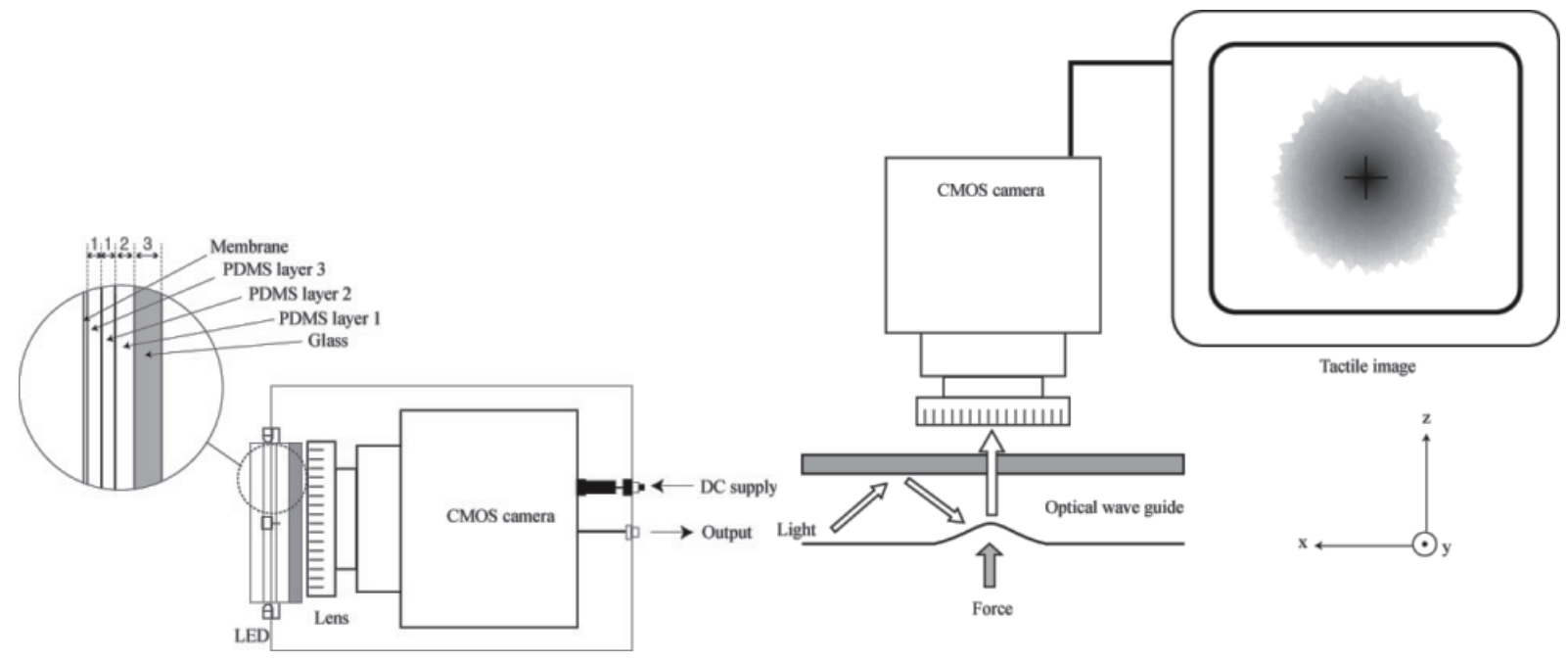

Fig. 2. Schematic of the optical tactile sensor.

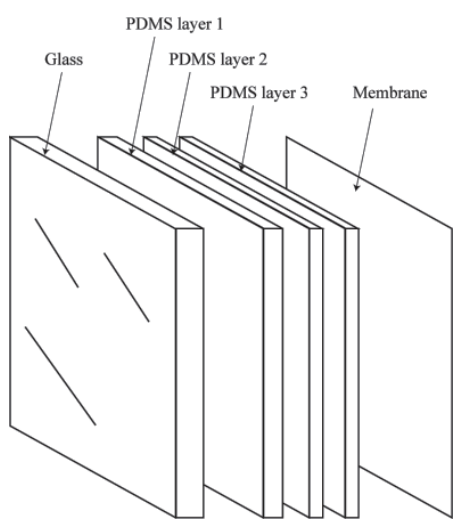

Fig. 3. Design of multilayer elastic waveguide.

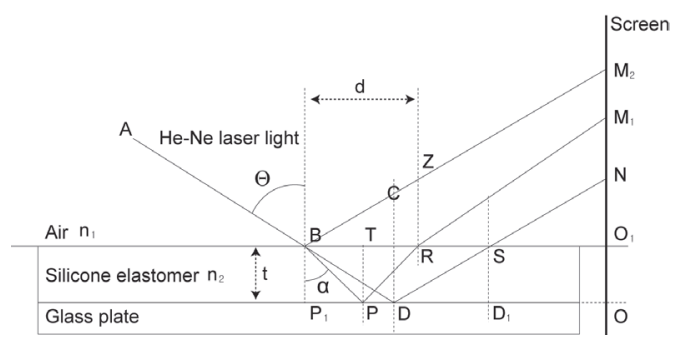

Fig. 4. Critical angle cone and acceptance angle in total internal reflection.

We should then get two spots appearing on the wall: one from the reflection on the surface of the liquid $\left(M_{2}\right)$ and one from the beam that refracts through the liquid and reflects from the glass $\left(M_{1}\right)$. If we assume the angle of incidence to be $\Theta$ and the angle of refraction as $\alpha$, then using Snell's law,

$$
n_{1} \sin \Theta=n_{2} \sin \alpha,
$$

where $n_{1}$ and $n_{2}$ are the refractive indices of air and the experimental liquid, respectively. From similar triangles in Fig. 4, one can easily see that

$$
\tan \Theta=O D / O N
$$

Using the triangle $B D S$, it can be shown from Fig. 4 that 


$$
B S=2 t \tan \Theta
$$

Similarly, from the triangle $B P R$, we have

$$
B R=2 t \tan \alpha .
$$

We also have

$$
\begin{gathered}
O_{1} S=O_{1} N \tan \Theta \\
O_{1} B=O_{1} M_{2} \tan \Theta .
\end{gathered}
$$

Therefore, from Eqs. (5) and (6),

$$
\begin{gathered}
O_{1} B-O_{1} S=\left(O_{1} M_{2}-O_{1} N\right) \tan \Theta, \\
B S=N M_{2} \tan \Theta .
\end{gathered}
$$

A comparison of Eqs. (3) and (8) gives

$$
N M_{2}=2 t
$$

Now from Eq. (4),

$$
\begin{aligned}
\tan \alpha & =B R / 2 t \\
& =\left(O_{1} B-O_{1} S\right) / 2 t \\
& =\left(O_{1} M_{2} \tan \Theta-O_{1} M_{1} \tan \Theta\right) / 2 t \\
& =\left(O_{1} M_{2}-O_{1} M_{1}\right) \tan \Theta / 2 t \\
& =M_{1} M_{2} \tan \Theta / 2 t .
\end{aligned}
$$

Using Eq. (2) for $\tan \Theta$ and Eq. (9) for $2 t$ gives

$$
\tan \alpha=M_{1} M_{2}(O D / O N) / N M_{2} .
$$

Now, by substituting equations, we obtain

$$
n_{1}=n_{2} \frac{\sin \left[\tan ^{-1}(O D / O N)\right]}{\sin \left\{\tan ^{-1}\left[\left(M_{2} M_{1} / N M_{2}\right) O D / O N\right]\right\}} .
$$

Note that there is a minimum necessary thickness of the PDMS for the points $M_{1}$ and $M_{2}$ to be solved. The experiment can be repeated for different angles of incidence and for different thicknesses of PDMS. 


\subsubsection{CMOS camera}

The high-performance CMOS camera is placed below an optical waveguide. A clear glass is placed between the CMOS camera and the optical waveguide to sustain an elastic optical waveguide without losing the camera resolution. The glass also acts as the bone and nail in a human finger. The digital imager is a monocooled CMOS with $65(\mathrm{H}) \times 65(\mathrm{~V}) \mu^{2}$ individual pixel size (FLEA2, Point Grey Research, British Columbia). The maximum lens resolution is $1392(\mathrm{H}) \times 1042(\mathrm{~V})$ and its angle of view is $60^{\circ}$.

\subsubsection{Internal light source}

The internal light source is a micro-LED (Unique-Leds, Newalla, OK) with a diameter of 1.8 $\mathrm{mm}$. There are four LEDs used to provide sufficient illumination to the optical waveguide.

\subsection{Tactile sensing principle}

The tactile sensor operates on the principle of TIR. ${ }^{(1)}$ Beams of light emitted from the light source to the edge of the optical waveguide are totally enclosed and reflected inside the optical waveguide. The tactile sensor uses the deformable properties of the waveguide as part of the transduction process. The light from a light source is injected into a waveguide from its edge. The innermost region of a waveguide carried the light, while preventing the light from leaking out of the silicone by reflecting the light within the boundaries of the silicone. When an object touches the optical waveguide and compresses, the deformed area causes the light to scatter, which is observed from the rear side of the transparent mirror. The concept is shown in Fig. 5.

Snell's law explains TIR. It states that the angle at which light reflects as it passes from one material to another depends on the refractive indices of the two materials - in this case, the elastic optical waveguide and air. Each medium is characterized by their complex refractive index $N=n-i k$. $N$ stands for the complex refractive index, $n$ is the real part, and $k$ is the imaginary part of the refractive index. $k$ is often denoted as the extinction coefficient. The index of refraction is the ratio of the velocity of light in a vacuum, $c$, to its velocity in a specific medium, $v$, as $n=c / v$. Light bends or refracts as it passes from a medium of one refractive index to a medium with a different refractive index. If a beam of light hits the interface of two

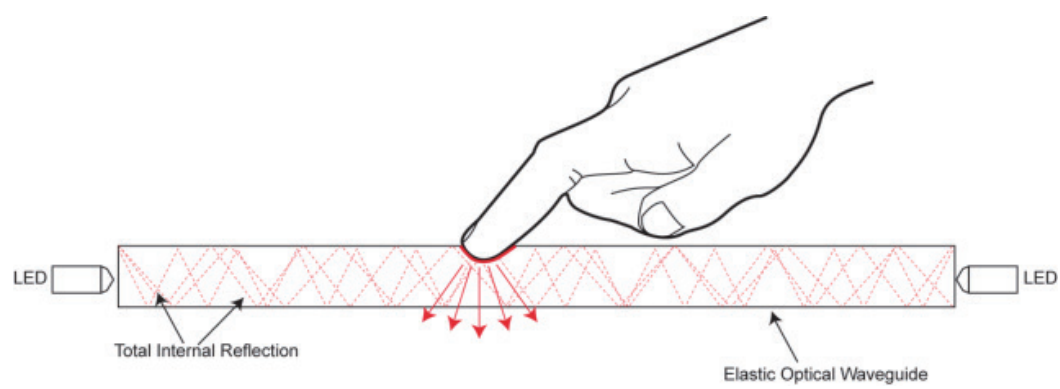

Fig. 5. (Color online) Tactile sensation concept using TIR. 
media, it will be partially reflected $(r)$ and partially transmitted (refracted) $(t)$. Let $n_{1}$ be the real part of the refractive index of medium 1 and $n_{2}$ that of medium 2. $\Theta_{1}$ and $\Theta_{2}$ are the angles between the surface normal and the incident and transmitted beams, respectively.

Snell's law determines the amount of light that bends as follows.

$$
n_{0} \sin \Theta_{0}=n_{1} \sin \Theta_{1}
$$

Increasing the angle of incidence over the critical angle results in TIR. Because the critical angle is achieved when $\Theta_{1}=90^{\circ}$, the critical angle $\Theta_{c}$ is calculated as

$$
\Theta_{c}=\sin ^{-1}\left(n_{0} / n_{1}\right) .
$$

Once the incidence angle is greater than the critical angle, $100 \%$ of the light reflects at the interface region. The principle of TIR governs the operation of the tactile sensor. This occurs because light travels at different speeds in different materials.

An imaginary cone of acceptance with an angle $\Theta_{a}$, determined by the critical angle, relates to a parameter called the numerical aperture $(N A)$ of the silicone elastomer. The acceptance cone angle constrains the light source transmittance angle for total internal refraction. $N A$ describes the light-gathering capability of the silicone elastomer. When a light ray is incident from a medium of refractive index $n_{0}$ to the silicone elastomer of index $n_{1}$, Snell's law at the medium silicone elastomer interface gives

$$
n_{0} \sin \Theta_{a}=n_{1} \sin \Theta_{r} .
$$

From Fig. 4 and using trigonometry, we get $\sin \Theta_{r}=\sin \left(90-\Theta_{c}\right)=\cos \Theta_{c}$, where $\Theta_{c}=$ $\sin ^{-1}\left(n_{0} / n_{1}\right)$ is the critical angle for TIR. After substituting, we obtain

$$
\left(n_{0} / n_{1}\right) \sin \Theta_{a}=\cos \Theta_{c} .
$$

By squaring both sides,

$$
\left(n_{0}^{2} / n_{1}^{2}\right) \sin ^{2} \Theta_{a}=\cos ^{2} \Theta_{c}=1-\sin ^{2} \Theta_{c}=1-n_{0}^{2} / n_{1}^{2} .
$$

Thus, the $N A$ is

$$
N A=n_{0} \sin \Theta_{a}=\sqrt{n_{1}^{2}-n_{0}^{2}},
$$

and the cone of acceptance angle is

$$
\Theta_{a}=\sin ^{-1}\left[\left(1 / n_{0}\right) \sqrt{n_{1}^{2}-n_{0}^{2}}\right] .
$$




\subsection{TIR in multiplayer PDMS}

The problem we are dealing with is the analysis of the condition of the TIR in multilayer PDMS. The basic design of the optical waveguide plate consists of three different densities of PDMS. Let us consider that PDMS is a nonabsorbing medium (i.e., $N_{2}, N_{3}, N_{4}, N_{5}$ are real, $N_{2}=$ $n_{2}, N_{3}=n_{3}, N_{4}=n_{4}, N_{5}=n_{5}$ ) on a semi-infinite substrate (this means that no light returns from the back surface of the substrate) and media 1 and 6 are air $\left(N_{1}=N_{6}=1\right)$. Light is incident from an absorption-free medium and strikes each layer of a stack of silicone forming the total waveguide plate. When incident light impinges upon the silicone surface, a portion is reflected, while the rest is transmitted to the other layer. Since each medium has a different refractive index, the incident angle changes as the light goes through a different medium. To totally reflect light in PDMS, the angle of all incident light into media 2 and 5 should be less than the critical angle. ${ }^{(12)}$ Below, the optimal cone of acceptance angle of the LED light source for TIR in multilayer PDMS is provided.

Assume that LED light sources are placed in the middle of multilayer PDMS. The lights of positive going strike media 2 and 3 and the lights of negative going strike media 4 and 5 . The critical and cone acceptance angles for the direct incidence to medium 2 are already shown in Fig. 4.

$$
\begin{gathered}
\Theta_{c_{2}}=\sin ^{-1}\left(n_{1} / n_{2}\right) \\
\Theta_{a_{2}}=\sin ^{-1}\left[\left(1 / n_{1}\right) \sqrt{n_{2}^{2}-n_{1}^{2}}\right]
\end{gathered}
$$

Another light incident to medium 2 is the light from medium 3. The refractive angle of light from media 3 to 2 should be less than the critical angle of $\Theta_{c_{1}}$ to maintain TIR in medium 2 . The critical angle of incident light from media 3 to 2 is as follows:

$$
\begin{gathered}
n_{2} \sin \Theta_{c_{2}}=n_{3} \sin \Theta_{c_{3}}, \\
\sin \Theta_{c_{2}}=\left(n_{2} / n_{3}\right) \sin \Theta_{c_{3}}=\left(n_{2} / n_{3}\right) \sin \left[\sin ^{-1}\left(n_{1} / n_{2}\right)\right]=n_{1} / n_{3}, \\
\Theta_{c_{3}}=\sin ^{-1}\left(n_{1} / n_{3}\right) .
\end{gathered}
$$

The cone acceptance angle is

$$
\begin{aligned}
n_{1} \sin \Theta_{a_{3}} & =n_{2} \sin \left(90-\Theta_{c_{3}}\right)=n_{2} \cos \Theta_{c_{3}}, \\
\Theta_{a_{3}} & =\sin ^{-1}\left[\left(1 / n_{0}\right) \sqrt{n_{3}^{2}-n_{0}^{2}}\right] .
\end{aligned}
$$

The analysis for lights of negative going follows the same procedure as that for lights of positive going. The light that directly strikes medium 5 has the following critical angle and cone acceptance angle: 


$$
\begin{gathered}
\Theta_{c_{5}}=\sin ^{-1}\left(n_{6} / n_{5}\right), \\
\Theta_{a_{5}}=\sin ^{-1}\left[\left(1 / n_{6}\right) \sqrt{n_{5}^{2}-n_{6}^{2}}\right] .
\end{gathered}
$$

The lights incident from media 4 to 5 have the following optical angles:

$$
\begin{gathered}
\Theta_{c_{4}}=\sin ^{-1}\left(n_{6} / n_{4}\right), \\
\Theta_{a_{4}}=\sin ^{-1}\left[\left(1 / n_{6}\right) \sqrt{n_{4}^{2}-n_{6}^{2}}\right] .
\end{gathered}
$$

Since the refractive index increases as the number of layers decreases, medium 2 has the smallest critical angle and largest cone acceptance angle. Therefore, if the light incidence angle is within the angle of $\Theta_{a_{2}}$, the light will be totally refracted in multilayer PDMS. The optical waveguide consists of three PDMS layers and the refractive indexes $n_{1}, n_{2}$, and $n_{3}$ measured are approximately $1.39,1.40$, and 1.41 , respectively. The outside of the waveguide is air and the refractive index of air, $n_{0}$, is 1 . Then, the light is negative going from the second layer. Thus, the critical angle for TIR is $\Theta_{c}=\sin ^{-1}(1 / 1.40)=45.58^{\circ}$ and the numerical aperture is $N A=\sqrt{1.40^{2}-1^{2}}=0.979$. Finally, the acceptance angle $\Theta_{a}$ of the imaginary cone is $\Theta_{a}=$ $\sin ^{-1}(0.979)=78.23$.

\subsection{Optical analysis of multilayer silicone}

If we obtain TIR light in PDMS, the response of an optical system to incident light can be analyzed. Let $E_{1}$ be the field vector of the incident beam from the LED light source. $E_{1}$ is split into the two components $E_{p}$ and $E_{s}$, which are parallel and perpendicular to the plane of incidence, respectively. Then, the amplitude refraction and transmission coefficients of parallel and perpendicular field vectors, $r_{p}, r_{s}$ and $t_{p}, t_{s}$ respectively, are given by Fresnel's equations. The Fresnel coefficients describe the ratios of the amplitudes of the respective beams to the corresponding amplitude of the incident beam.

$$
\begin{aligned}
& r_{p}=\frac{E_{p, r}}{E_{p, 1}}=\frac{N_{2} \cos \Theta_{1}-N_{1} \cos \Theta_{2}}{N_{2} \cos \Theta_{1}+N_{1} \cos \Theta_{2}} \\
& r_{s}=\frac{E_{s, r}}{E_{s, 1}}=\frac{N_{1} \cos \Theta_{1}-N_{2} \cos \Theta_{2}}{N_{1} \cos \Theta_{1}+N_{2} \cos \Theta_{2}} \\
& t_{p}=\frac{E_{p, t}}{E_{p, 1}}=\frac{2 N_{1} \cos \Theta_{1}}{N_{1} \cos \Theta_{1}+N_{1} \cos \Theta_{2}} \\
& t_{s}=\frac{E_{s, t}}{E_{s, 1}}=\frac{2 N_{1} \cos \Theta_{1}}{N_{1} \cos \Theta_{1}+N_{2} \cos \Theta_{2}}
\end{aligned}
$$


In the general case, these coefficients are complex numbers. As intensities are proportional to the square of the complex field amplitudes, the corresponding intensity reflection coefficients are given by $R_{12}=r_{12} r_{12}^{*}$ and so on, where $r^{*}$ denotes the complex conjugate. The notation $R_{i j}$ means the reflection coefficient for light incident from medium 1 at the interface to medium 2 . The transmission coefficient $T_{12}$ is given by $1-R_{12}$. Note that $T_{12}$ owing to its definition by the Poynting vector is not equal to $t_{12} t_{12}^{*}$, but

$$
T_{12}=1-R_{12}=\frac{N_{2} \cos \Theta_{2}}{N_{1} \cos \Theta_{1}} t_{12} t_{12}^{*}
$$

Owing to the multiple reflectances at the internal interfaces, part of the incident light intensity traverses the thin film several times corresponding to an increase in effective optical path length. Therefore, we obtain an analytical dependence that differs significantly from the wellknown Lambert-beer law.

$$
I(x)=I_{o} \exp (-\alpha x)
$$

The Lambert-Beer law describes the attenuation of light travelling through isotropic, homogeneous matter. $I(x)$ is the remaining intensity at position $x, I_{o}$ is the incident intensity at position $x=0$, and $\alpha$ is the absorption coefficient. $\alpha$ is related to the imaginary part of the refractive index (the coefficient $k$ ) of the medium as follows:

$$
\alpha=4 \pi k / \lambda \text {. }
$$

It is common to define the reflectance $R$ of the sample as the ratio of the reflected intensity $I_{r}$ to the incident intensity $I_{i}, R=I_{r} / I_{i}$, and the transmittance $T$ as $T=I_{t} / I_{i}$, the absorptance $A$ as $A=I_{a} / I_{i}$, and the optical scatter $S$ as the ratio of the diffusely scattered intensity to the incident intensity, $S=I_{S} / I_{i}$. The reflectance and transmittance are often also named reflectivity and transitivity, respectively. Energy conservation requires

$$
R+T+A+S=1 .
$$

In many practical applications, particularly those considered here, the optical scatter is negligible and the equation simplifies to

$$
R+T+A=1 .
$$

For a PDMS optical sensor, it is not appropriate to use the Lambert-Beer law because multiple reflections in PDMS should be considered, as indicated in Fig. 5. The reflected intensity is composed of an infinite number of individual, multiply reflected beams. In the case of a nonabsorbing layer (i.e., $N_{2}, N_{3}, N_{4}, N_{5}$ are real, $N_{2}=n_{2}, N_{3}=n_{3}, N_{4}=n_{4}, N_{5}=n_{5}$ ) on a semiinfinite substrate (this means that no light returns from the back surface of the substrate), we can write the reflectance $R$ as 


$$
R=R_{\text {medium } 2}+R_{\text {medium } 3}+R_{\text {medium } 4}+R_{\text {medium } 5},
$$

where

$$
\begin{aligned}
R_{\text {medium } 2} & =R_{12}+T_{12} R_{21} T_{23} R_{23}+T_{12} T_{23}\left(R_{21} R_{23}\right)^{2}+\cdots \\
& =R_{12}+T_{12} T_{23} \sum_{j=1}^{\infty}\left(R_{12} R_{23}\right)^{j-1}=R_{12}+\frac{T_{12} T_{23}}{1-R_{21} R_{23}}, \\
R_{\text {medium } 3} & =R_{13}+T_{13} T_{32} R_{32} T_{34} R_{34}+T_{13} T_{32} T_{34}\left(R_{32} R_{34}\right)^{2}+\cdots \\
& =R_{13}+T_{13} T_{32} T_{34} \sum_{j=1}^{\infty}\left(R_{32} R_{34}\right)^{j-1}=R_{13}+\frac{T_{13} T_{32} T_{34}}{1-R_{32} R_{34}}, \\
R_{\text {medium } 4} & =R_{14}+T_{14} T_{43} R_{43} T_{45} R_{45}+T_{14} T_{43} T_{45}\left(R_{43} R_{45}\right)^{2}+\cdots \\
& =R_{14}+T_{14} T_{43} T_{45} \sum_{j=1}^{\infty}\left(R_{43} R_{45}\right)^{j-1}=R_{14}+\frac{T_{14} T_{43} T_{45}}{1-R_{43} R_{45}}, \\
R_{\text {medium } 5} & =R_{15}+T_{15} T_{54} R_{54} R_{56}+T_{15} T_{54}\left(R_{54} R_{56}\right)^{2}+\cdots \\
& =R_{15}+T_{15} T_{54} \sum_{j=1}^{\infty}\left(R_{54} R_{56}\right)^{j-1}=R_{15}+\frac{T_{15} T_{54}}{1-R_{54} R_{56}} .
\end{aligned}
$$

The transmittance is given by $T=1-R$. It should be mentioned here that the summation of the intensities corresponds to the incoherent superposition of individual light beams.

\subsection{Mechanical analysis of multilayer silicone}

The active sensing area of the elasticity sensor is capped with PDMS layers. The contact between a rigid indenter and a flat PDMS is of our interest. The shape of the indenter may be a spherical, conical, or cylindrical flat punch, or may even take the form of a uniformly applied pressure. All such phenomena are usually referred to as "Hertzian contact"." (13) The most wellknown scenario is the contact between a rigid sphere and a flat surface where Hertz found that the radius of the circle of contact, $a$, is related to the indenter force $F$, the effective radius of curvature, $R$, and the elastic properties of the materials by

$$
a^{3}=\frac{4}{3} \frac{k F R}{E}
$$

where $k$ is an elastic mismatch factor given by

$$
k=\frac{9}{16}\left[\left(1-v^{2}\right)+\frac{E}{E^{\prime}}\left(1-v^{\prime 2}\right)\right]
$$

and $R=\left(\frac{1}{R_{1}}+\frac{1}{R_{2}}\right)^{-1}$ 
In the equation above, $E, v$ and $E^{\prime}, v^{\prime}$ are the Young's moduli and Poisson's ratios of the specimen and indenter, respectively.

Hertz also found the maximum stress as

$$
p_{\max }=\frac{3 F}{2 \pi a^{2}} .
$$

Thus, the predicted stress profile is given as

$$
p(x, y)=p_{\max }\left[1-\left(\frac{r}{a}\right)^{2}\right]^{\frac{1}{2}},
$$

where $r=\left[x^{2}+y^{2}\right]^{1 / 2}$.

Figure 6 shows the Hertzian contact when the ball shape indenter is in contact with a PDMS surface. Let us assume that the stainless steel of the indenter has a modulus of $200 \mathrm{GPa}$ and a Poisson ratio of 0.30 . If the indenter has a diameter of $5 \mathrm{~mm}$ and the two are being held together by $2 \mathrm{~N}$ force, this gives the effective radius

$$
R=(1 / 5 \mathrm{~mm}+1 / \infty)^{-1}=5 \mathrm{~mm}
$$

and the effective modulus

$$
E^{0}=\left[\frac{\left(1-0.3^{2}\right)}{200 \times 10^{3} \mathrm{MPa}}+\frac{\left(1-0.4^{2}\right)}{1 /(1 / 0.14+1 / 0.08+1 / 0.034) \mathrm{MPa}}\right]^{-1}=0.0243 \mathrm{MPa} .
$$

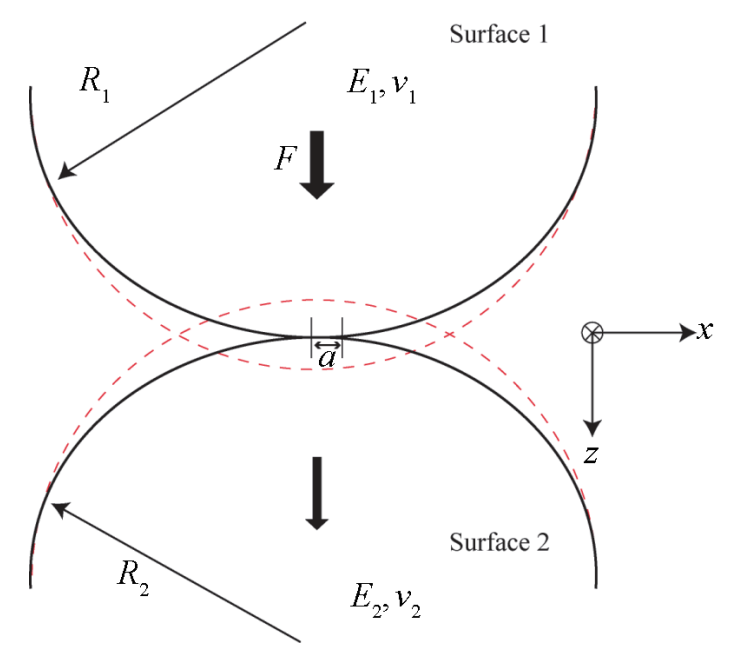

Fig. 6. (Color online) Hertzian contact diagram. $F$ is the force applied to the other. $R_{1}, E_{1}$, and $v_{1}$ and $R_{2}, E_{2}$, and $v_{2}$ are the radius, Young's modulus, and Poisson's ratio of surfaces 1 and 2, respectively. $a$ is half the length of the contact patch, which is defined by the other variables. 
The indenter will deform by

$$
a=\left(\frac{3 \times 2 \mathrm{~N} \times 0.005 \mathrm{~m}}{4 \times 0.0243 \mathrm{MPa}}\right)^{\frac{1}{3}}=0.1029 \mathrm{~mm} .
$$

The maximum pressure that can be applied is then

$$
p_{\max }=\frac{3 \times 2 \mathrm{~N}}{2 \pi \times 0.1029^{2}}=90.2256 .
$$

The stress profile of the contact surface is given in Eq. (48). The simulation under a different normal force is given in Fig. 7.

The Hertzian contact surface is calculated by changing the indenter's modulus and radius. The result is shown in Fig. 8. From the figure, we observe that, as the modulus of the indenter increases, the sum of the Hertzian contact stress profile converges. The same situation occurs when the radius of the indenter increases. To remove the effective variation from a different indenter, the sufficiently large modulus and radius of the indenter should be considered.

\subsection{Normal and shear force detection}

To define the force direction, a Cartesian coordinate frame is added to the figure. If the optical waveguide accepts a three-axis force, it is deformed in both compressive and shearing directions. Because the light scatters in the contact area, the gray-scale value of the tactile image acquired by the CCD camera distributes as a Gaussian function, in which the gray-scale intensity is the highest at the centroid and decreases with increasing distance from the centroid.

Since the gray-scale light spot $R(x, y)$ of the tactile image is proportional to the contact pressure $p(x, y)$ caused by the contact between the optical waveguide and an object, it can be expressed as

$$
p(x, y)=C(R(x, y))
$$
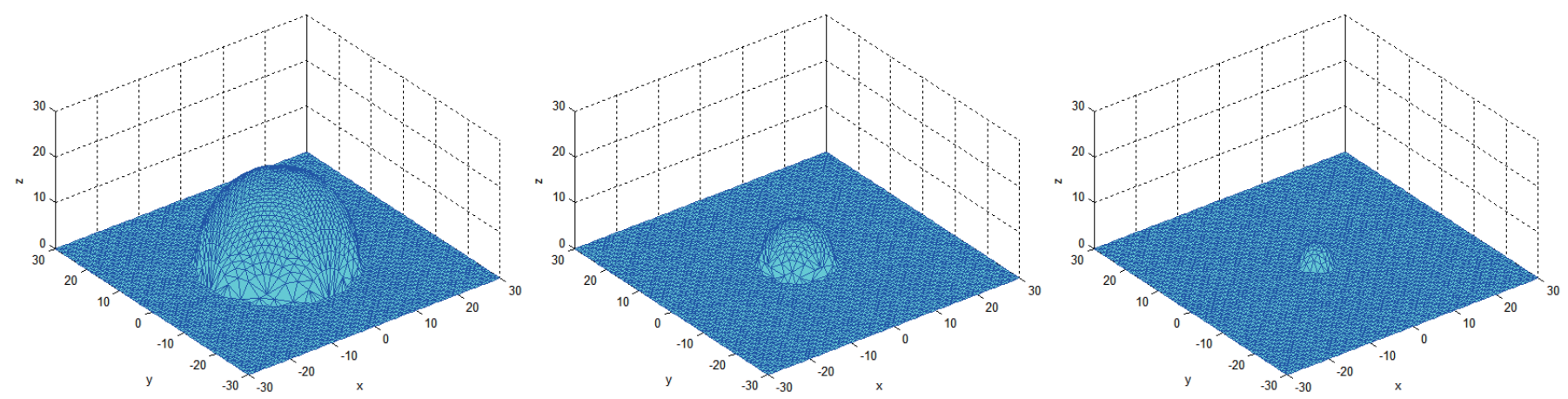

Fig. 7. (Color online) Surface stress profile predicted by Hertzian contact theory for spherical indenter (diameter: $2 \mathrm{~mm}$ ) with $0.5,1$, and $1.5 \mathrm{~N}$. The spacing between mesh points is $0.03 \mathrm{~mm}$. 


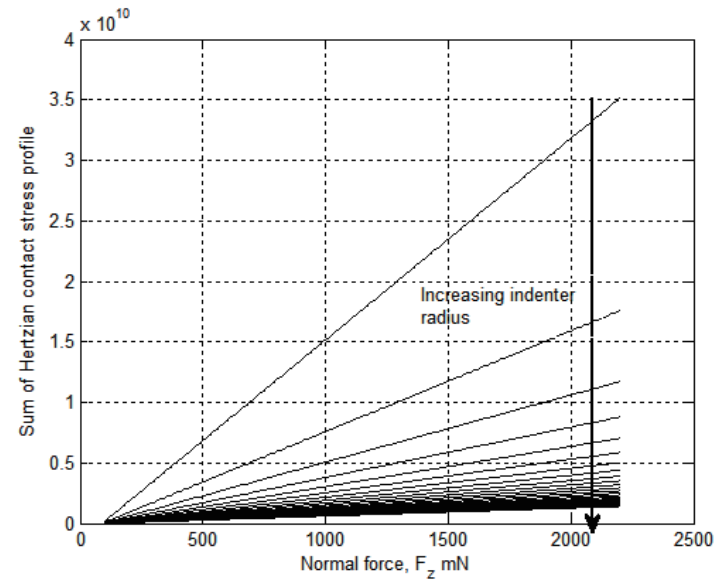

Fig. 8. Sum of Hertzian contact stress profile on the contact surface versus normal force under different indenter radii.

where $C$ is the conversion factor. If $S$ is designated as the contact area of silicone and an object, the vertical force $F_{z}$ is obtained by integrating the pressure over the contact area as

$$
F_{z}=\int_{S} p(x, y) d S
$$

If we substituted Eq. (53) into Eq. (54), then the vertical force is $F_{z}=\int_{S} p(x, y) d S$ $=\int_{S} C(g(x, y)) d S \equiv C(G)$, where the integration of $g(x, y)$ over the contact area is denoted as $G$.

To formulate the horizontal force vectors $F_{x}$ and $F_{y}$, the $x$ and $y$ coordinates of the centroid $\left(X_{c}, Y_{c}\right)$ are calculated as

$$
\begin{aligned}
& X_{c}=\int_{S} g(x, y) x d S / \int_{S} g(x, y) d S, \\
& Y_{c}=\int_{S} g(x, y) y d S / \int_{S} g(x, y) d S .
\end{aligned}
$$

Since the shearing force induces the strain in the optical waveguide, it moves in the horizontal direction. The $x$ and $y$ components of the movement are denoted as $u_{x}$ and $u_{y}$ and expressed as

$$
\begin{aligned}
& u_{x}=X_{c}^{(t)}-X_{c}^{(t-1)}, \\
& u_{y}=Y_{c}^{(t)}-Y_{c}^{(t-1)},
\end{aligned}
$$

where $(t)$ and $(t-1)$ represent current and prior steps, respectively. If friction between the optical waveguide and the contact object is ignored, the $x$ - and $y$-directional forces $F_{x}$ and $F_{y}$ are calculated as 


$$
\begin{aligned}
& F_{x}=K_{x} u_{x}, \\
& F_{y}=K_{y} u_{y},
\end{aligned}
$$

where $K_{x}$ and $K_{y}$ are the $x$ - and $y$-directional spring constants of PDMS, respectively.

\subsection{D rendering tactile image}

The local regions in a tactile image correspond to illuminated patches of a piecewise continuous surface $z(x, y)$. The measured brightness $R(x, y)$ will depend on the material properties of the surface, the orientation of the surface at the coordinates $(x, y)$, and the direction and strength of illumination. The surface orientation is described by the components of the surface gradient in the $x$ and $y$ directions, i.e., $p=\partial z / \partial x$ and $p=\partial z / \partial y$. In this section, we recover the surface $z(x, y)$ from the image $R(x, y)$.

The common assumptions are that the surface has an ideal Lambertian reflectance and a constant albedo. ${ }^{(18)}$ If a surface exhibits Lambertian reflectance, light falling on it is scattered such that the apparent brightness of the surface to an observer is the same regardless of the observer's angle of view. This means that the surface luminance is isotropic. Then, the radiosity at a point $(x, y)$ on the surface of the object is given by

$$
B(x, y)=\rho(x, y) N(x, y) \cdot L,
$$

where $\rho(x, y)$ is the surface albedo, $N$ is the surface normal vector, and $L$ is the light source vector. Assuming that the camera response is linear to the surface radiosity $B(x, y)$, the reflectance of each pixel can be written as

$$
R(x, y)=k B(x, y)=k \rho(x, y) N(x, y) \cdot L
$$

If we assume that the sensor surface is the Lambert surface with the albedo $\rho(x, y)$ as 1 and the camera constant $k$ as 1 , then $N(x, y)$ can be determined as

$$
R(x, y)=N(x, y) \cdot L=|N(x, y)||L| \cos \Theta .
$$

The light is totally reflected in PDMS, and the surface normal vector points in the same direction as the light source vector. The surface normal vector may be written as $N(x, y)=(-p,-q, 1)^{\mathrm{T}}$, where $p=\partial z / \partial x$ and $p=\partial z / \partial x$. For a light source vector, we can similarly write the light source direction as $L=\left(-p_{l},-q_{l}, 1\right)^{\mathrm{T}}$ and $p=p_{l}$ and $q=q_{l}$. Thereby, it makes $V$ a vector containing elements related to both the scene lighting and the camera, $V=$ $N(x, y) \cdot L$.

Once the field of normal vector and its light source vector, we can easily recover the $z$-coordinate for each point in the mesh. If we assume that the unit normal vector $\hat{V}$ at some point $(x, y)$ is $(a(x, y), b(x, y), c(x, y))$ and that $z(x, y)$ is the function of the surface, it is easy to see that $\frac{\partial z}{\partial x}=\frac{-a(x, y)}{c(x, y)}$ and $\frac{\partial z}{\partial y}=\frac{-b(x, y)}{c(x, y)}$. If we assume that the $z$-value at some reference point 
$p_{o}$ is 0 , the $z$-value at any other point $p_{d}$ will be a linear integral of $\left(\frac{\partial z}{\partial x}, \frac{\partial z}{\partial y}\right)$ along the path from $p_{o}$ to $p_{d}$. The following Fig. 9 represents the 2D tactile image of $1 \mathrm{~N}$ small tip loading and its $3 \mathrm{D}$ rendering image.

\section{Stiffness Measurement}

In this section, a nonrigid point matching algorithm is introduced for the reconstruction of sensed object properties. The stiffness of an object is estimated by optimally minimizing the spline energy function. A nonrigid point matching method is derived to calculate the displacement field and spline function iteratively, which significantly enhances the efficiency and stability.

\subsection{Problem formation}

Let $S=\left\{s_{1}, s_{2}, \ldots, s_{I}\right\}$ be a set of control points of a tactile image and $T=\left\{t_{1}, t_{2}, \ldots, t_{J}\right\}$ be a set of control points of another tactile image under different compression ratios. The individual control point $s_{i}$ is represented as a two-dimensional homogeneous coordinate vector $\left(1, s_{i x}, s_{i y}\right)$. In a matching problem, one-to-one matching is desired, but in general, one-to-one matching is not possible because of outliers. To handle this problem, two point sets are augmented to $\hat{S}=\left\{s_{1}, s_{2}, \ldots, s_{I}\right.$, nil $\}$ and $\hat{T}=\left\{t_{1}, t_{2}, \ldots, t_{I}\right.$, nil $\}$ by introducing a dummy or a nil point. Then, common points can be matched one-to-one and outliers can be matched with a dummy point nil. Every point has its adjacent points called neighbors. For a given point $s_{i} \in \hat{S}$, one can select adjacent points $a_{x}^{s_{i}}, x=1, \ldots, X$, and $X$ is the total number of adjacent points of $s_{i}$. Similarly, for a point $t_{j} \in \hat{T}$, the adjacent points are $a_{y}^{t_{j}}, y=1, \ldots, Y$ and $Y$ is the total number of adjacent points of $t_{j}$.

\subsection{Strain fields from point displacement}

The coordinates $s_{i}$ and $t_{j}$ are related by deformation that occurs between the acquisitions of the two 3D images. To provide a continuous displacement field for strain analysis, we apply a smoothing algorithm to $s_{i}$ and $t_{j}$. The smoothing algorithm can be obtained by the

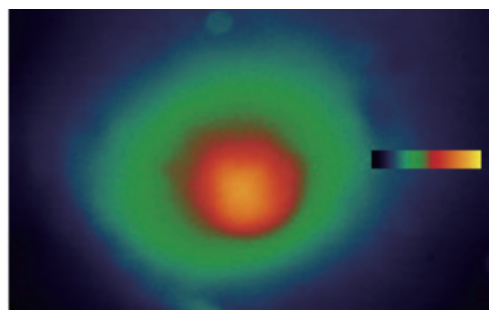

(a)

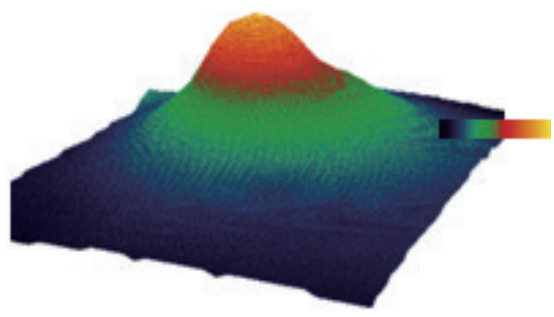

(b)

Fig. 9. (Color online) (a) 2D tactile image and (b) 3D rendering image based on Lambertian surface assumption. 
transformation of point displacement. Given a finite set of correspondences between points on two images, one can proceed to estimate the plane transformation $T: \mathfrak{R}^{3} \rightarrow \mathfrak{R}^{3}$ that may be used to map arbitrary points from one image to the other. The specified correspondences consist of a small number of control points and $T$ extends the correspondences to arbitrary points.

We need to choose $T$ from a suitable family of transformations. A standard choice is the affine model, i.e,

$$
T(x)=A x+o,
$$

with some matrix $A$ and the translational offset vector $o$ parameterizing the set of all allowed transformations. Then, the least-squares solution $\hat{T}=(\hat{A}, \hat{o})$ is obtained as

$$
\begin{gathered}
\hat{o}=\frac{1}{n} \sum_{i=1}^{n}\left(s_{i}-t_{i}\right), \\
\hat{A}=\left(Q^{+} P\right)^{t} .
\end{gathered}
$$

There are many different methods that can be used to smooth data. In this study, we mostly use the thin plate spline (TPS) model, which is commonly used for representing flexible coordinate transformations. Bookstein found it to be highly effective for modeling changes in biological forms. Powell applied the TPS model to recover transformations between curves. The TPS is the 2D generalization of the cubic spline. In its regularized form, which is discussed below, the TPS model includes the affine model as a special case. We will now provide some background information on the TPS model.

We start with the $1 \mathrm{D}$ interpolation problem. Let $v_{i}$ denote the target function values at the corresponding locations $p_{i}=\left(x_{i}, y_{i}\right)$ in the plane, with $i=1,2, \ldots, n$. In particular, we will set $v_{i}$ equal to $x_{i}^{\prime}$ and $y_{i}^{\prime}$ in turn to obtain one continuous transformation for each coordinate. We assume that the locations $\left(x_{i}, y_{i}\right)$ are all different and not collinear. The TPS interpolant $f(x, y)$ minimizes the bending energy

$$
I_{f}=\iiint_{3}\left\{\left(\frac{\partial^{2} f}{\partial x^{2}}\right)^{2}+\left(\frac{\partial^{2} f}{\partial y^{2}}\right)^{2}+\left(\frac{\partial^{2} f}{\partial z^{2}}\right)^{2}+2\left[\left(\frac{\partial^{2} f}{\partial x \partial y}\right)^{2}+\left(\frac{\partial^{2} f}{\partial x \partial z}\right)^{2}+\left(\frac{\partial^{2} f}{\partial y \partial z}\right)^{2}\right]\right\} d x d y d z
$$

and has the form

$$
f(x, y, z)=a_{1}+a_{x} x+a_{y} y+a_{z} z+\sum_{i=1}^{n} w_{i} U\left(\left\|\left(x_{i}, y_{i}, z_{i}\right)-(x, y, z)\right\|\right),
$$

where the kernel function $U(r)$ is defined by $U(r)=r^{2} \log r^{2}$ and $U(0)=0$ as usual. In order for $f(x, y)$ to have square integrable second derivatives, we require that $\sum_{i=1}^{n} w_{i}=0$ and $\sum_{i=1}^{n} w_{i} x_{i}=\sum_{i=1}^{n} w_{i} y_{i}=0$. 
Together with the interpolation conditions, $f\left(x_{i}, y_{i}\right)=v_{i}$, this yields a linear system for the TPS coefficients:

$$
\left(\begin{array}{cc}
K & P \\
P^{\mathrm{T}} & 0
\end{array}\right)\left(\begin{array}{l}
w \\
a
\end{array}\right)=\left(\begin{array}{c}
w \\
0
\end{array}\right)
$$

where $k_{i j}=U\left(\left\|\left(x_{i}, y_{i}\right)-\left(x_{j}, y_{j}\right)\right\|\right)$, the $i$ th row of $P$ is $\left(1, x_{i}, y_{i}\right), w$ and $v$ are column vectors formed from $w_{i}$ and $v_{i}$, respectively, and $a$ is the column vector with the elements $a_{1}, a_{x}$, and $a_{y}$. We will denote the $(n+3) \times(n+3)$ matrix of this system by $L$. $L$ is nonsingular and we can find the solution by inverting $L$. If we denote the upper left $n \times n$ block of $L^{-1}$ by $A$, then it can be shown that

$$
I_{f} \propto v^{\mathrm{T}} A v=w^{\mathrm{T}} K w .
$$

When there is noise in the specified value $v_{i}$, one may wish to relax the exact interpolation requirement by regularization. This is accomplished by minimizing

$$
H[f]=\sum_{i=1}^{n}\left[v_{i}-f\left(x_{i}, y_{i}\right)\right]^{2}+\lambda I_{f}
$$

The regularization parameter $\lambda$, a positive scalar, controls the amount of smoothing; the limiting case of $\lambda=0$ decreases to exact interpolation.

We use two separate TPS functions to model a coordinate transformation,

$$
T(x, y, z)=\left(f_{x}(x, y, z), f_{y}(x, y, z), f_{z}(x, y, z)\right),
$$

which yields a displacement field that maps any position in the first image to its interpolated location in the second image. The nonlinear Lagrangian strain tensor components were then determined in the imaging plane from Ref. 14.

$$
\begin{gathered}
e_{x x}=\frac{\partial f_{x}(x . y)}{\partial x}+\frac{1}{2}\left[\left(\frac{\partial f_{x}(x, y)}{\partial x}\right)^{3}+\left(\frac{\partial f_{y}(x, y)}{\partial x}\right)^{3}+\left(\frac{\partial f_{z}(x, y)}{\partial x}\right)^{3}\right] \\
e_{y y}=\frac{\partial f_{x}(x . y)}{\partial y}+\frac{1}{2}\left[\left(\frac{\partial f_{x}(x, y)}{\partial y}\right)^{3}+\left(\frac{\partial f_{y}(x, y)}{\partial y}\right)^{3}+\left(\frac{\partial f_{z}(x, y)}{\partial y}\right)^{3}\right] \\
e_{z z}=\frac{\partial f_{x}(x . y)}{\partial z}+\frac{1}{2}\left[\left(\frac{\partial f_{x}(x, y)}{\partial z}\right)^{3}+\left(\frac{\partial f_{y}(x, y)}{\partial z}\right)^{3}+\left(\frac{\partial f_{z}(x, y)}{\partial z}\right)^{3}\right] \\
e_{x y}=\frac{1}{2}\left(\frac{\partial f_{x}(x, y)}{\partial y}+\frac{\partial f_{y}(x, y)}{\partial x}\right)+\frac{1}{2}\left[\left(\frac{\partial f_{x}(x, y)}{\partial x}\right)\left(\frac{\partial f_{x}(x, y)}{\partial y}\right)+\left(\frac{\partial f_{y}(x, y)}{\partial x}\right)\left(\frac{\partial f_{y}(x, y)}{\partial y}\right)\right] \\
e_{x z}=\frac{1}{2}\left(\frac{\partial f_{x}(x, y)}{\partial z}+\frac{\partial f_{z}(x, y)}{\partial x}\right)+\frac{1}{2}\left[\left(\frac{\partial f_{x}(x, y)}{\partial x}\right)\left(\frac{\partial f_{x}(x, y)}{\partial z}\right)+\left(\frac{\partial f_{z}(x, y)}{\partial x}\right)\left(\frac{\partial f_{z}(x, y)}{\partial z}\right)\right]
\end{gathered}
$$




\subsection{Measurement of elastic properties}

According to the Hertz contact theory, a contact of two spheres of Young's moduli $E_{f}$ and $E_{o}$ generates a circular contact area $A$. The relative displacements of the two spheres are $w_{f}$ and $w_{o}$, and the relationship between the relative displacements and Young's modulus is

$$
w_{o} E_{o}=w_{f} E_{f}
$$

As the Young's modulus of an object to touch, $E_{o}$, is larger, the displacement $w_{o}$ is smaller. Similarly, as the Young's modulus of the waveguide, $E_{f}$, is larger, the displacement $w_{f}$ is smaller. With a fixed value $E_{o}$, if the modulus of the waveguide, $E_{f}$, increases, the estimated stress should decrease since the displacement $w_{f}$ decreases. In the optical tactile sensor method, the normal force applied to an object is obtained through a linear curve fit. The strain calculation for point distribution is also from the tactile image. Thus, the computed strain and estimated normal force increases and decreases simultaneously and finally we can obtain the fixed $E_{o}$ accordingly.

To determine the apparent elastic properties of each sample from this uniaxial loading configuration, the strain components were averaged along the $x$ and $y$ directions at each axial coordinate $z$, to yield the average strain $\bar{e}_{z z}$. Given the applied normal stress component $\left(S_{z z}\right.$, second Piola-Kirchoff stress) acting on the loading surfaces, the apparent Young's modulus $E$ was then determined as

$$
E=S_{z z} / \bar{e}_{z z}
$$

\section{Experimental Results}

\subsection{Experimental conditions}

We developed the loading machine shown in Fig. 10 that includes an $x$-stage, a $z$-stage, and a force/torque gauge (Mecmesin, West Sussex, UK) to detect the sensing characteristics of forces. The force gauge has a probe to measure force and can detect force ranging from 0 to $50 \mathrm{~N}$ with a resolution of $0.001 \mathrm{~N}$. The system is composed of a tactile sensor, a loading machine, image processing software, and a computer.

\subsection{Normal force detection test}

To find the applied normal force from the tactile image, the relationship between the contact force $F_{z}$ and the calibrated gray-scale light spot intensity $R(x, y)$ is evaluated. Since the camera is an 8-bit digital imager, each $R(x, y)$ value is quantified by a gray-scale level and has a minimum of 0 and a maximum of $2^{8}=256$. In this experiment, a sharp tip with $2 \mathrm{~mm}$ radius attached to the force/torque gauge is used to contact the tactile sensor. To validate the normal force detection, after the initial load of $0 \mathrm{mN}$, the normal force is increased in a stepwise manner. When the applied force reaches the maximum force, in this experiment, $2 \mathrm{~N}$, the applied normal force decreases in a stepwise fashion until it returns to $0 \mathrm{~N}$. The resulting 


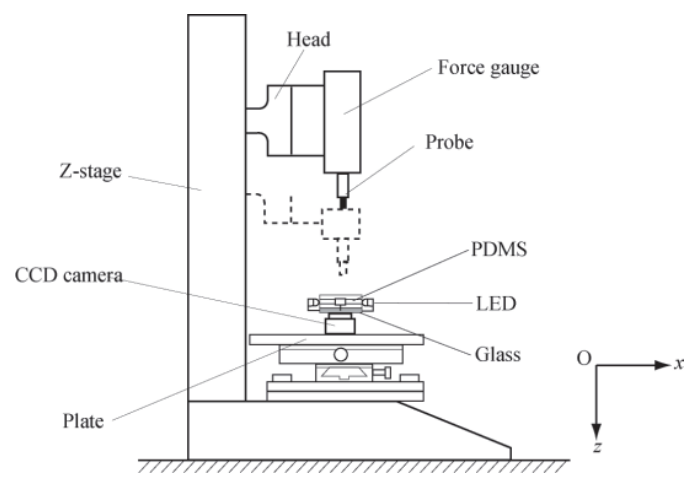

Fig. 10. Tactile data processing system.

light spot is captured by the tactile sensor and the corresponding contact force is measured by the force gauge. A pixel corresponds to pressure and the total pixel is measured to determine pressure characteristics. Figure 11 shows a series of tactile images. The original image is enhanced by the linear filter. Figure 12 shows the relationship between the integrated grayscale value and the applied normal force. As indicated in this figure, the relationship between the integrated gray-scale value and the applied force is linear.

The fitting curve was achieved as shown in Fig. 13. The curve approximately represents the monotonic increasing relationship between the contact force $F_{z}$ and the integrated gray-scale value $G$ of the tactile image as follows.

$$
C(G)=1.75 \times 10^{-5} G-114.56
$$

The slope yields the effective spring constant $k$ for the optical tactile sensor.

\subsection{Modulus estimation experiment}

A Versaflex CL2000X (GLS, McHenry, Illinois) soft polymer with the known Young's modulus of about $103 \mathrm{kPa}$ was used to validate the measurement methods developed in this study. For this experiment, the Varsaflex sample was cut to cubic size so that the top surface area of the sample was less than the optical waveguide area. The optical tactile sensor had a compressed polymer sample and the compression ratio was slowly increased. The obtained tactile images were rendered to the 3D shape and $N$ points were sampled from the shape boundary, also referred to as the shape distribution. We sample the surface of the shape with roughly uniform spacing. The sampling method used throughout this study is from Ref. 15. It is a fast and efficient random sampling method. Figure 14 shows the point distributions of $0.8 \mathrm{~N}$ and $1.0 \mathrm{~N}$ 3D tactile images before registration method. The point distribution after registration is shown in Fig. 15. Figure 16 shows the experimental results. The solid line represents the gold standard of the CL200X modulus and the open circle represents the measurements of such a modulus. The modulus estimated using the optical tactile sensor was within $23 \%$ compared with the gold standard. 


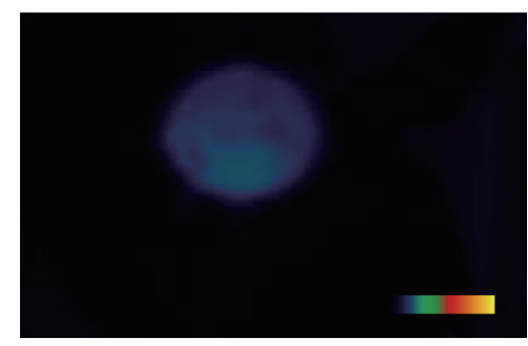

(a)

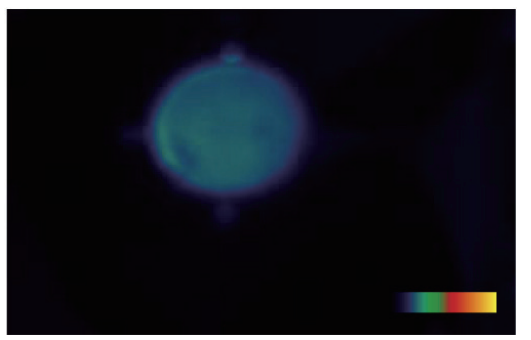

(b)

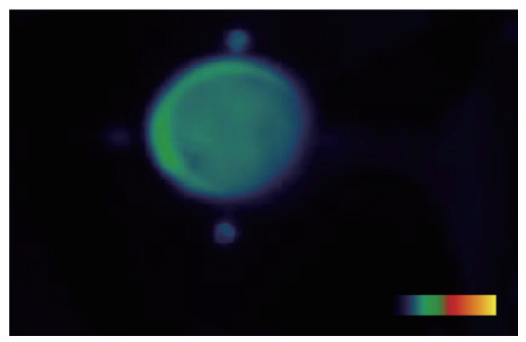

(c)

Fig. 11. (Color online) Tactile images with (a) 537, (b) 966, and (c) $1503 \mathrm{mN}$ normal forces.

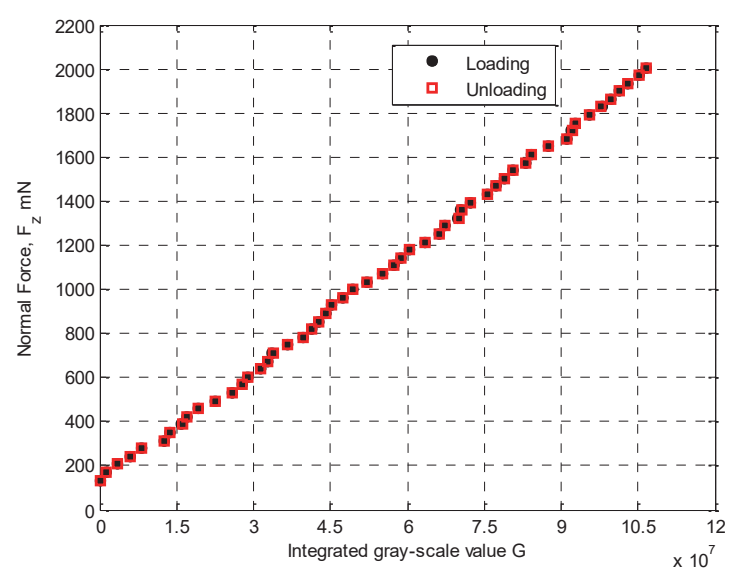

Fig. 12. (Color online) Relationship between normal force and integrated gray-scale value.

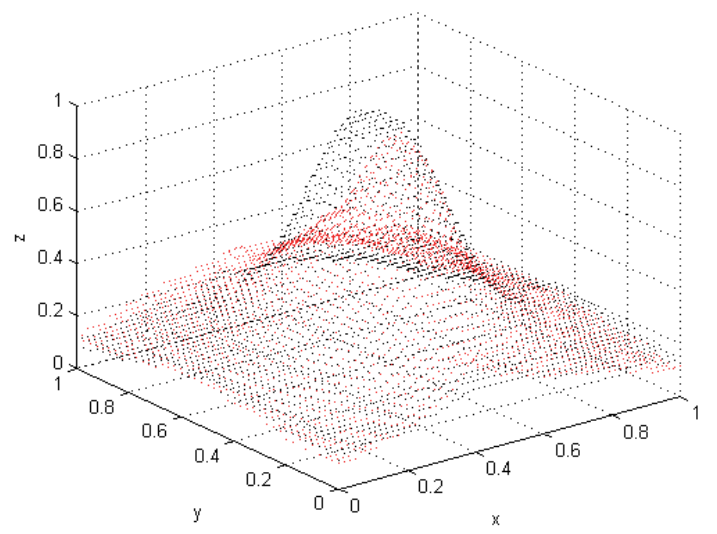

Fig. 14. (Color online) Control points from 3D tactile images under $0.8 \mathrm{~N}$ (red) and $1 \mathrm{~N}$ (black) compressions before registration.

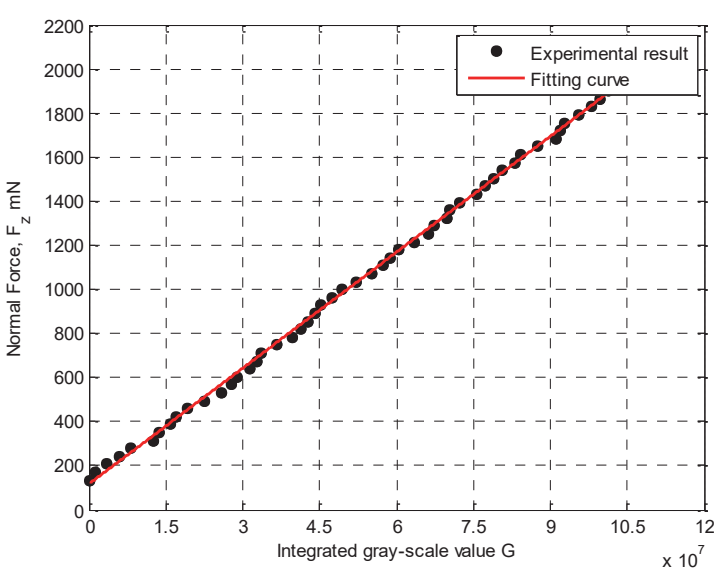

Fig. 13. (Color online) Curve fitting of loading and unloading data.

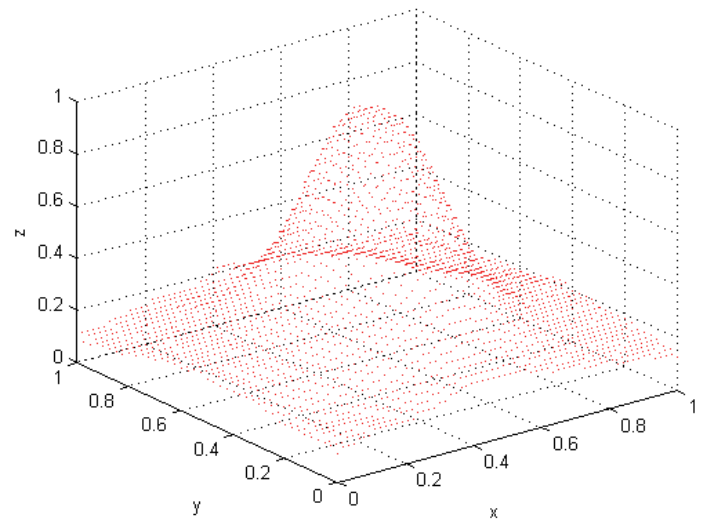

Fig. 15. (Color online) Control points from 3D tactile images under $0.8 \mathrm{~N}$ (red) and $1 \mathrm{~N}$ (black) compressions after registration. 


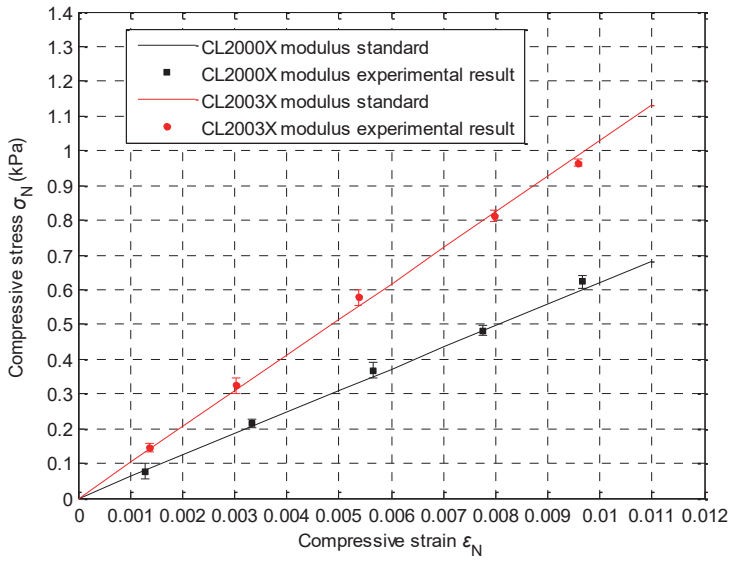

Fig. 16. (Color online) Experimental results of polymer sample modulus estimation.

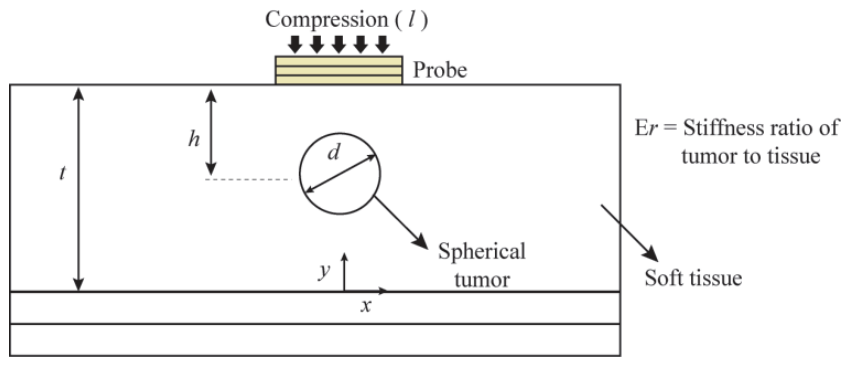

Fig. 17. (Color online) Transverse section of a tissue and tumor, labeled with input parameters for finite element model.

\section{Application of Tactile Sensing in Tumor Detection}

\subsection{Detection of tumor using a computational method}

Optical tactile sensing is a new approach to detecting objects embedded in biological tissues. This approach, in comparison with other methods, is noninvasive. We develop algorithms that are used to extract the features of objects that have been imaged. Three-dimensional analysis leads to a novel method of predicting the characteristics of a tumor; it can be directly incorporated with tactile sensing in artificial palpation. The finite element method (FEM) is needed to prove properties such as the shape, size, depth, and location of a tumor. Figure 17 shows a transverse section of a tissue and tumor, labeled with input parameters. The input parameters are as follows.

1. Tissue loading (compression of the tissue surface), $l$

2. Tissue thickness, $t$

3. Tumor diameter, $d$

4. Tumor depth, $h$

5. Stiffness ratio of tumor to tissue, $E_{r}$

6. Tumor shape, $s$

The aim is to investigate the effects of an object embedded in a biological tissue associated with the application of loading on the tissue. The stress distribution is obtained from the tactile image. A graph is extracted by plotting the stress magnitude on a specific axis. Then, the changes in stress response relative to variations in input parameters are investigated.

\subsection{Inverse problems}

Although it is predicted that the results of the forward approach on VI-A can show the existence of tumors, they do not provide detailed information on the tumor characteristics. In this section, one technique for solving inverse problems using an artificial neural network (ANN) is the use of an inverse method for forward results. Figure 18 shows a flowchart of this problem. The 

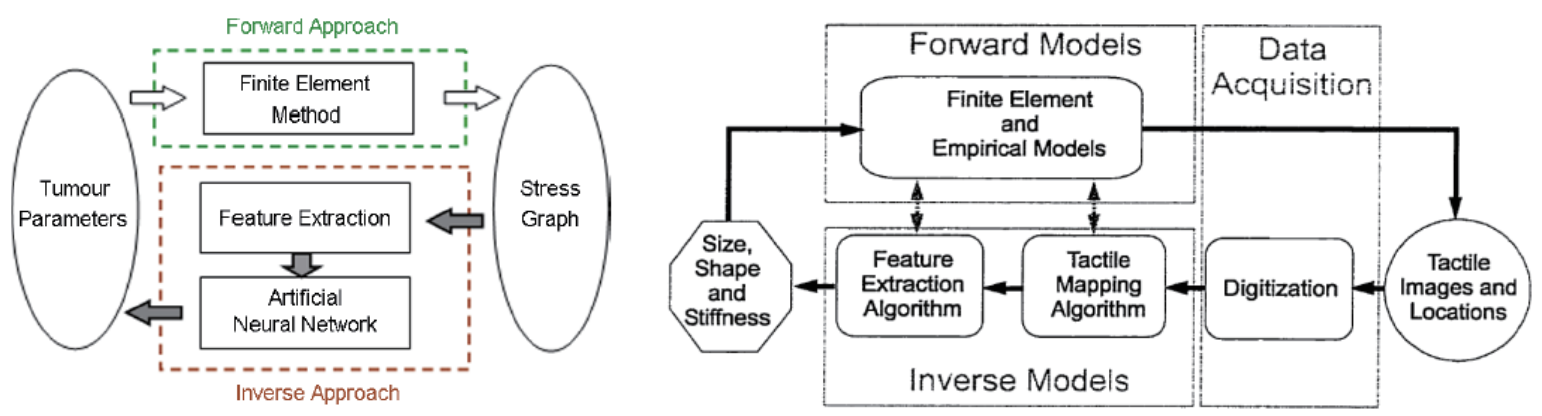

Fig. 18. (Color online) Flowchart of forward and inverse approaches.

first part is the data acquisition and construction of tactile maps using the FEM. In the second part, we require an inverse model that takes a tactile map as input and produces the size and shape of the mass that has been imaged as output.

ANNs are characterized in principle by a network topology, a connection pattern, neural activation properties, a training strategy, and the ability to process data. Neural networks have been employed to determine the tumor characteristics, where stress graphs were the inputs of the neural network and the tumor characteristics were the desired outputs.

\subsection{Experimental results}

\subsubsection{Measurement accuracy as a function of the inclusion size}

The realistic breast tissue phantoms shown with embedded hard nodules have been prepared. This model was made of a Zerdine composite having a Young's modulus of $4 \mathrm{kPa}$ with an array of hard spherical inclusions embedded at different depths in the model. The phantom thickness was measured to be $20 \mathrm{~mm}$, and inclusions were placed $5 \mathrm{~mm}$ below the upper surface of the phantoms. The inclusion was a ball made by Play-Doh (Play-Doh, Hasbro, Newport, UK). The inclusion sizes were 12, 8 , and $4 \mathrm{~mm}$. The tactile images are obtained under the same force of 2 N. Figure 19 shows the experimental concept.

Because light scatters on the contact area, the gray-scale value of the contact image acquired by the CCD camera distributes as a bell shape, in which the gray-scale intensity is the highest at the centroid and decreases with increasing distance from the centroid of the deformed area. To compare the tactile image, the horizontal tactile data through the centroid is obtained and the Gaussian fitting model is used to describe the shape of the tactile data (Fig. 20). From this curve, we obtain the following Gaussian function and, from this equation, we obtain the pressure intensity distribution.

$$
P=a e^{-x^{2} / 2 \sigma^{2}}
$$

The amplitude of each Gaussian function is defined by $a_{1}=2323, a_{2}=137.06$, and $a_{3}=77.07$. Moreover, the variance is defined by $\sigma_{1}^{2}=99.96, \sigma_{2}^{2}=98.92$, and $\sigma_{3}^{2}=75.40$. From this data, we can clearly see the difference between three data. 


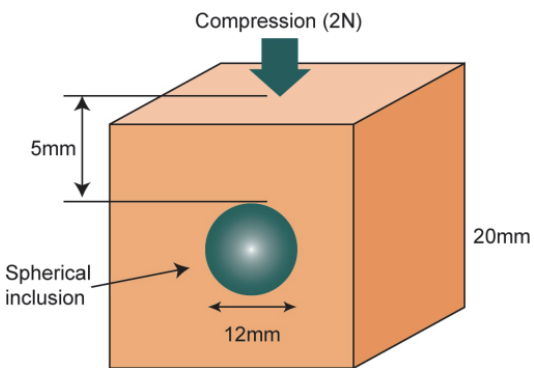

(a)

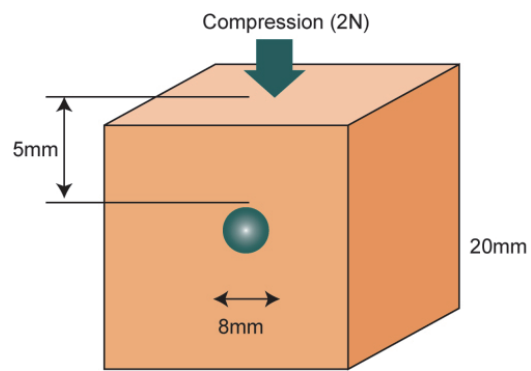

(b)

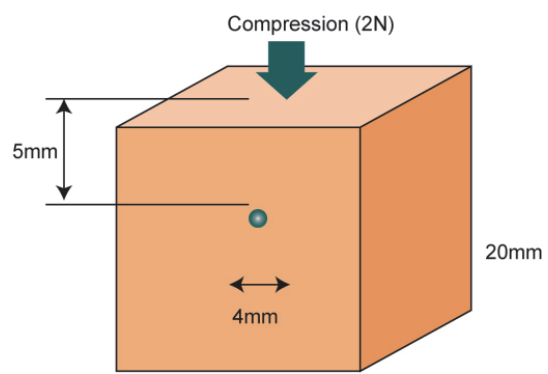

(c)

Fig. 19. (Color online) Example of the strain experienced as a function of inclusion size. (a) Tissue sample with 12 mm inclusion. (b) Tissue sample with $8 \mathrm{~mm}$ inclusion. (c) Tissue sample with $4 \mathrm{~mm}$ inclusion.

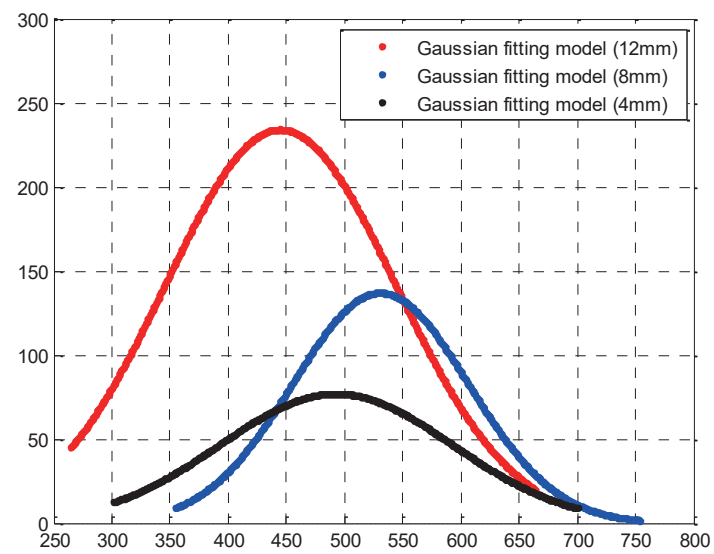

Fig. 20. (Color online) Three Gaussian fitting models depending on inclusion size.

\subsubsection{Measurement accuracy as function of difference in inclusion}

In this experiment, the phantoms had an inclusion of 8-mm-diameter Play-Doh and rubber. Typically, the rubber has a much higher elastic modulus than Play-Doh. The total phantom thickness was $20 \mathrm{~mm}$, and the base material had a Young's modulus of $4 \mathrm{kPa}$. The inclusions were placed $5 \mathrm{~mm}$ below the upper surface of the phantoms. The tactile images are generated under 2.35 N. Figure 21 shows the experimental concept.

Figure 22 shows a Gaussian fitting model. The rubber tactile data shows $a_{1}=175.20$ with $\sigma_{1}^{2}=80.25$ and the Play-Doh tactile data shows $a_{1}=81.62$ with $\sigma_{1}^{2}=98.6$.

\section{Discussion}

In this study, we have experimented and demonstrated our method using the breast phantom models embedding hard nodules instead of breast cancer. Therefore, in future works, we will conduct experiments to detect breast cancer. The proposed sensing strategy measures the Young's modulus of polymer samples within $23 \%$ error. In our future work, we will study methods that can reduce this error rate. 


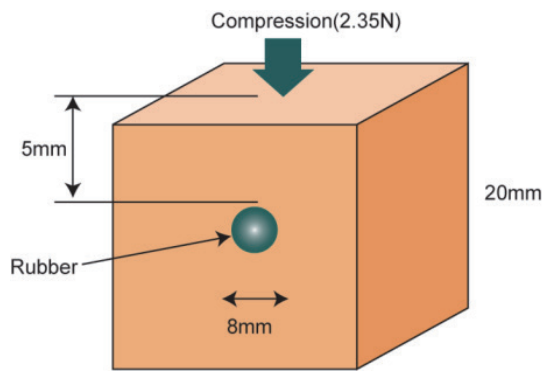

(a)

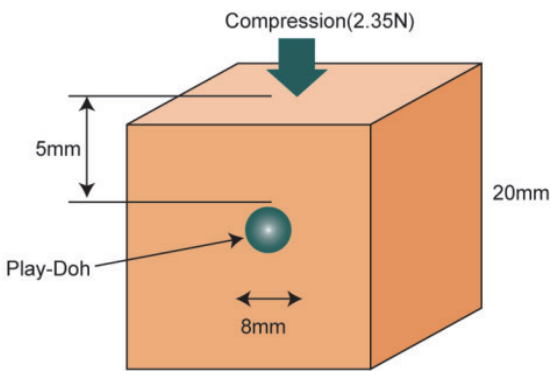

(b)

Fig. 21. (Color online) Example of the strain experienced as a function of inclusion material. (a) Tissue sample with $8 \mathrm{~mm}$ rubber inclusion. (b) Tissue sample with $8 \mathrm{~mm}$ Play-Doh inclusion.

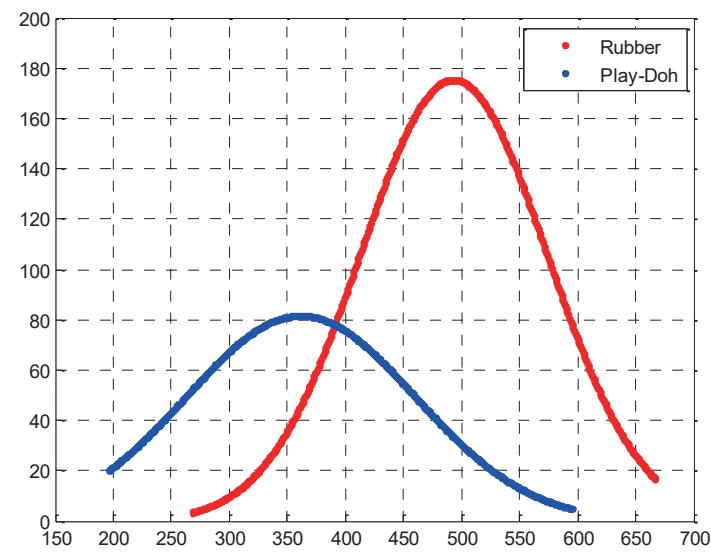

Fig. 22. (Color online) Three Gaussian fitting models depending on inclusion material.

\section{Conclusions}

A novel tactile sensor capable of measuring the material constants of sensed objects has been fabricated and demonstrated in the current study. The sensor operates on the principle of TIR within an optical waveguide. Since the waveguide is surrounded by air, which has a lower refractive index than the waveguide, the incident light directed into the waveguide remains contained within it. When an object compresses the waveguide, the contact area of the waveguide deforms and causes the light to scatter. Since the scattered light is directly captured by a CCD camera, the tactile resolution of the proposed sensor is based on the resolution of the camera. The normal force is detected from the integrated gray-scale values of bright pixels emitted from the deformed area of the optical waveguide. Experiments were conducted to demonstrate the ability of the proposed sensing strategy to measure the Young's modulus of polymer samples within $23 \%$ error.

\section{Acknowledgments}

This research was supported by the Keimyung University Research Grant of 2017. 


\section{References}

1 F. Kamangar, G. M. Dores, and W. F. Anderson: J. Clin. Oncol. 24 (2006) 2137.

2 A. Jemal, R. Siegel, E. Ward, Y. Hao, J. Xu, and M. J. Thun: Cancer J. Clinicians 59 (2009) 225.

3 H. Shojaku, H. Seto, H. Iwai, S. Kitazawa, W. Fukushima, and K. Saito: Radiation Med. 26 (2008) 362.

4 A. Fenster and D. B. Downey: IEEE Eng. Med. Biol. Mag. 15 (1996) 41.

5 H. Degani, V. Gusis, D. Weinstein, S. Fields, and S. Strano: Nat. Med. 3 (1997) 780.

6 F. L. Bookstein: Morphometric Tools for Landmark Data: Geometry and Biology (Cambridge Univ. Press, London/New York, 1991) p. 383.

7 M. J. D. Powell: Computational Techniques and Applications: CTAC95, eds. RL May and AK Easton (World Scientific, Singapore, 1996) p. 43.

8 C. R. Gentle: J. Biomed. Eng. 10 (1988) 124.

9 H. W. Goodson, III and D. H. Moore II: JAMA 283 (2000) 1687.

10 T. A. Krouskop, T. M. Wheeler, F. Kallel, B. S. Garra, and T. Hall: Ultrasonic Imaging 20 (1998) 260.

11 T. Ratanachaikanont: J. Med. Assoc. Thailand Chotmaihet thangphaet 88 (2005) 505.

12 J. Ophir, I. Cespedes, H. Ponnekanti, Y. Yazdi, and X. Li: Ultrasonic Imaging 13 (1991) 111.

13 L. Gao, K. J. Parker, R. M. Lerner, and S. F. Levinson: Ultrasound Med. Biol. 22 (1996) 959.

14 J. Rogowska, N. A. Patel, J. G. Fujimoto, and M. E. Brezinski: Heart 90 (2004) 556.

15 A. Vinckier and G. Semenza: FEBS Lett. 430 (1998) 12.

16 H. Rivaz, E. Boctor, P. Foroughi, R. Zellars, G. Fichtinger, and G. Hager: IEEE Trans. Med. Imaging 27 (2008) 1373.

17 A. T. Stavros, D. Thickman, C. L. Rapp, M. A. Dennis, S. H. Parker, and G. A. Sisney: Radiology 196 (1995) 123.

18 J. A. Coakley: Encyclopedia of the Atmosphere, eds. J. Holton and J. Curry (Academic Press, 2003) pp. 19141923.

\section{About the Authors}

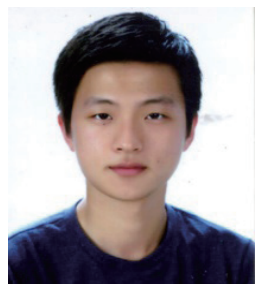

Su Yang received his B.S. degree from Keimyung University, Korea, in 2016. Currently, he is a graduate student of School of Medicine at Keimyung University, Korea. Before starting his graduate study, he researched about computer-aided diagnosis and machine learning. His research interests are in computer-aided diagnosis, machine learning, and optical sensors.

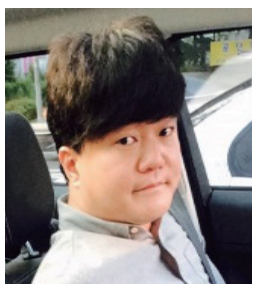

Jong-Ha Lee received his B.S. degree in electronics engineering in 2000 from Inha University, Incheon, Korea, M.S. degree in electrical engineering in 2005 from New York University, Brooklyn, New York, USA, and Ph.D. degree in electrical engineering from Temple University, Philadelphia, PA, USA. He was with Samsung Advanced Institute of Technology as a Research Staff Member. He is currently an assistant professor of the Department of Biomedical Engineering, School of Medicine Keimyung University, Daegu, Korea. His current research interests include tactile sensation imaging for tissue characterization, computer-aided diagnosis, medical image analysis, pattern recognition, and machine learning. 